\title{
Structure of wedge-induced oblique detonation in acetylene-oxygen-argon mixtures
}

Cite as: Phys. Fluids 31, 026108 (2019); https://doi.org/10.1063/1.5086235

Submitted: 19 December 2018 . Accepted: 27 January 2019 . Published Online: 15 February 2019

Yishen Fang (方宜申), Yuhang Zhang (张宇航) (D) Xi Deng (邓希), and Honghui Teng (滕宏辉) (D)
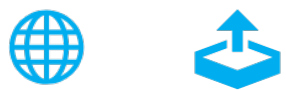

\section{ARTICLES YOU MAY BE INTERESTED IN}

Turbulence structure of a counter-flowing wall jet

Physics of Fluids 31, 025110 (2019); https://doi.org/10.1063/1.5082550

Numerical study of viscous effects on centreline shock reflection in axisymmetric flow Physics of Fluids 31, 026105 (2019); https://doi.org/10.1063/1.5085267

On the non-equilibrium models for subfilter scalar variance in large eddy simulation of turbulent mixing and combustion

Physics of Fluids 31, 025112 (2019); https://doi.org/10.1063/1.5066228

PHYSICS TODAY WHITEPAPERS
ADVANCED LIGHT CURE ADHESIVES

Take a closer look at what these

environmentally friendly adhesive systems can do
READ NOW

PRESENTED BY Q MASTERBOND: 


\title{
Structure of wedge-induced oblique detonation in acetylene-oxygen-argon mixtures
}

\author{
Cite as: Phys. Fluids 31, 026108 (2019); doi: 10.1063/1.5086235 \\ Submitted: 19 December 2018 - Accepted: 27 January 2019 • \\ Published Online: 15 February 2019
}

\begin{abstract}
Yishen Fang (方宜申), ${ }^{1,2}$ Yuhang Zhang (张宇航), ${ }^{3}$ (D) Xi Deng (邓希), ${ }^{4}$ and Honghui Teng (滕宏辉) ${ }^{3, a)}$ (D)

\author{
AFFILIATIONS \\ 'State Key Laboratory of High Temperature Gas Dynamics, Institute of Mechanic, Chinese Academy of Sciences, \\ Beijing 100190, China \\ ${ }^{2}$ School of Engineering Sciences, University of Chinese Academy of Sciences, Beijing 100049, China \\ ${ }^{3}$ School of Aerospace Engineering, Beijing Institute of Technology, Beijing 100081, China \\ 4Department of Mechanical Engineering, Tokyo Institute of Technology, Tokyo 152-8550, Japan
}

a) Author to whom correspondence should be addressed: hhteng@bit.edu.ch

\begin{abstract}
Stoichiometric acetylene-oxygen mixtures diluted by argon are widely used in normal detonation waves but have not yet been used in oblique detonation waves (ODWs). The present study simulates ODWs in acetylene-oxygen-argon mixtures, with a dilution ratio $\Phi=50 \%-90 \%$, using the reactive Euler equations with a detailed chemistry model, and describes the characteristics of acetylene ODWs. Similar to ODWs in hydrogen-air mixtures, the transition from the oblique shock wave to ODW may be either abrupt or smooth and the effects of $\Phi$ are investigated with a variable incident Mach number $M_{0}$. When $M_{0}$ changes between 8 and 10, the transition is abrupt in the case of $\Phi=50 \%$, while it is smooth in the case of $\Phi=90 \%$. In the case of $\Phi=70 \%$, a high $M_{0}$ corresponds to a smooth transition, while a low $\mathrm{M}_{0}$ corresponds to an abrupt transition. A further study on the initiation mechanism was performed by comparing the numerical initiation length with the length obtained via the constant-volume combustion calculation, which demonstrated that the initiation is kinetic-controlled in the cases of $\Phi=70 \%$ and $90 \%$, but wave-controlled in the case of $\Phi=50 \%$ with $M_{0}$ below 8.5. Moreover, an initiation structure featured by two-shock in the product was observed for the first time, and its formation mechanism is qualitatively discussed and compared to hydrogen ODWs.
\end{abstract}

Published under license by AIP Publishing. https://doi.org/10.1063/1.5086235

\section{INTRODUCTION}

In premixed combustible mixtures, there are two types of combustion waves: deflagration and detonation. Detonation propagates supersonically at high speed is the scale of $10^{3} \mathrm{~m} / \mathrm{s}$ since detonation is ignited by a strong leading shock, which differs from deflagration by diffusion effects. Traditionally, detonation research is useful to prevent loss by strong shock, such as catastrophic explosions and super-knock phenomena in automotive internal combustion engines. However, the idea to utilize detonation in engines attracts increasing attention, benefiting from the high thermal cycle efficiency of detonation combustion. Therefore, detonation-based engines become an important direction of the new-concept propulsion system in aeronautics and astronautics areas. ${ }^{7-4}$
An oblique detonation engine (ODE) is a detonation-based engine and also known as Shcramjet (Shock-induced combustion ramjet). It is applicable to air-breathing hypersonic propulsion, such as Scramjet (Supersonic combustion ramjet) ${ }^{5}$ but based on the oblique detonation wave (ODW). To facilitate practical engines, a fundamental understanding of ODW structure and instability are necessary to achieve standing and stable detonation in supersonic inflow. In early studies, e.g., Ref. 6, ODW was simplified as an oblique shock wave (OSW) coupled with an instant post-shock heat release. Later studies $^{7,8}$ demonstrated that for the wedge-induced ODW, there is a nonreactive OSW before the formation of the ODW surface. The OSW-ODW transition can be viewed as an initiation of ODW, which may generate various structures. . $^{9-13}$ However, several studies on the ODW surface instability ${ }^{14-20}$ have 
been reported, illustrating and discussing the appearance and evolution of fine-scale structures.

Based on the feature of the OSW-ODW transition, two types of transition structures can be observed, as shown in Fig. 1. One type is abrupt transition and has been studied widely; here, the main OSW and ODW are connected by a multi-wave point, as shown in Fig. 1(a). The other type is smooth transition in which the multi-wave point is replaced by a continuous curved shock, 7,10,11 as shown in Fig. 1(b). The abrupt type usually corresponds to a low $\mathrm{M}_{0}$ (incident Mach number), and the smooth type corresponds to a high $\mathrm{M}_{0}$; however, realistic structures beneath the multi-wave point or curved shock are more complicated than shown in Fig. 1. For the abrupt transition of Fig. 1(a), the secondary ODW may be replaced by a compression wave. However, the smooth transition must appear with a compression wave rather than with secondary ODW, but OSW in the product may appear. It has also been reported that decreasing $M_{0}$ results in various shock configurations, in which the transient process may play an important role. ${ }^{21-23}$

Due to the lack of deep knowledge of ODW initiation, and given certain inflow conditions, prior to simulation, it cannot be predicted whether a compression wave or secondary ODW forms. Because detonation waves are sensitive to the properties of combustible mixtures, as studied in normal and rotating detonation studies, ${ }^{24-27}$ ODWs in different fuels should be the fundamentals of the initiation structure study. A primary test employs a two-step induction-reaction global kinetic model ${ }^{28,29}$ to replace an oversimplified onestep irreversible heat release. Several novel phenomena have been observed because the two-step model has more parameters to model different mixtures. Based on detailed chemistry models, recent numerical studies have addressed the performance of ODWs in hydrogen-air mixtures, i.e., $\mathrm{H}_{2}-\mathrm{O}_{2}-\mathrm{N}_{2}$, and the effects of $\mathrm{M}_{0},{ }^{21}$ inflow inhomogeneity, ${ }^{30,31}$ and initiation features $^{32}$ have been further discussed.

In this study, the structure of wedge-induced ODW in acetylene-oxygen-argon mixtures, i.e., $\mathrm{C}_{2} \mathrm{H}_{2}-\mathrm{O}_{2}-\mathrm{Ar}$, was simulated and studied based on a detailed chemistry model.

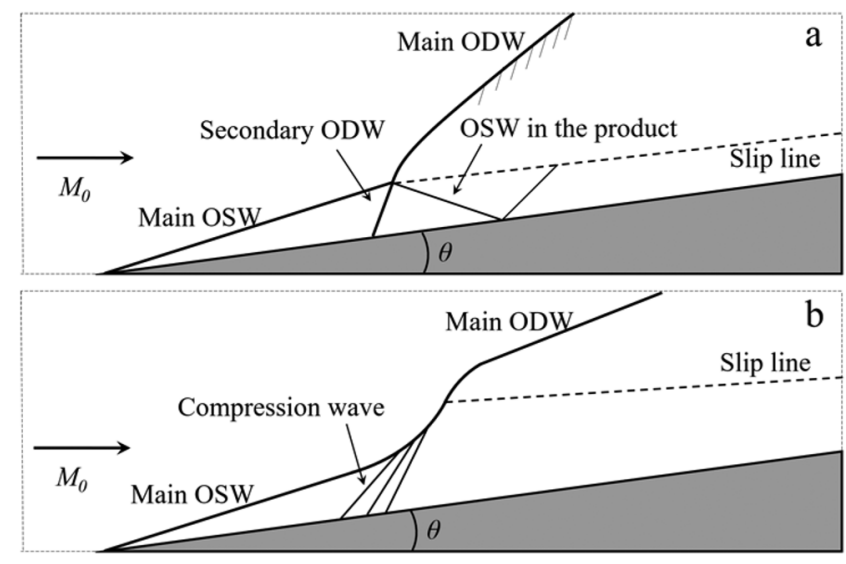

FIG. 1. Schematic of ODWs with abrupt (a) and smooth (b) transitions
$\mathrm{C}_{2} \mathrm{H}_{2}-\mathrm{O}_{2}-\mathrm{Ar}$ is a widely used type of fuel in normal detonation studies ${ }^{33-35}$ because it is easy to be initiated and has a regular cellular pattern under low pressure. On the ODW, experimental studies induced by conical shock conical have been performed by launching spherical projectiles. ${ }^{36,37}$ To investigate the structure of ODW, this study uses a 2D semi-infinite wedge, which provides a simple oblique shock-induced detonation in comparison to a recent study of $\mathrm{H}_{2}$-air mixtures. ${ }^{32}$ Effects of the dilatation ratio $\Phi$ on both the transition structure and initiation mechanism were investigated with variable $\mathrm{M}_{0}$, and a distinct two-oblique-shock structure was observed and analyzed.

\section{PHYSICAL AND MATHEMATICAL MODELS}

A schematic of this simulation study is presented in Fig. 2. For a high $\mathrm{M}_{0}$, causing a high post-shock temperature behind the OSW, an exothermic chemical reaction may lead to ODW initiation. The coordinate is rotated to the direction along the wedge surface and the Cartesian grid in the rectangular domain (enclosed by the dashed line in Fig. 2) is aligned with the wedge surface.

Previous results ${ }^{9,38}$ have demonstrated that both the viscosity and the boundary layer exert little effect on the overall ODW structure, in contrast to supersonic combustion. ${ }^{39-41}$ Then, most of the previous studies have been based on the inviscid assumption, and the reactive Euler equations have been used as governing equations to model the ODW flow field

$$
\frac{\partial \mathbf{U}}{\partial \mathrm{t}}+\frac{\partial \mathbf{F}}{\partial x}+\frac{\partial \mathbf{G}}{\partial y}=\mathbf{S}
$$

where

$$
\mathbf{U}=\left(\begin{array}{c}
\rho_{1} \\
\vdots \\
\rho_{n} \\
\rho u \\
\rho v \\
e
\end{array}\right), \mathbf{F}=\left(\begin{array}{c}
\rho_{1} u \\
\vdots \\
\rho_{n} u \\
\rho u^{2} \\
\rho u v \\
(e+\rho) u
\end{array}\right), \mathbf{G}=\left(\begin{array}{c}
\rho_{1} v \\
\vdots \\
\rho_{n} v \\
\rho u v \\
\rho v^{2}+p \\
(e+\rho) v
\end{array}\right), \mathbf{S}=\left(\begin{array}{c}
\omega_{1} \\
\vdots \\
\omega_{n} \\
0 \\
0 \\
0
\end{array}\right) .
$$

The total density and total energy are calculated by

$$
\rho=\sum_{i=1}^{n} \rho_{i},
$$

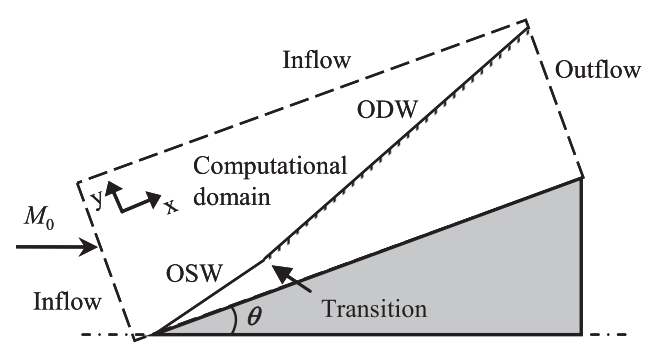

FIG. 2. Schematic of the computational settings. 


$$
e=\rho h-p+\frac{1}{2} \rho\left(u^{2}+v^{2}\right)
$$

where specific enthalpy can be written as $h=\sum_{i=1}^{n} \rho_{i} h_{i} / \rho$ with $h_{i}$ obtained from the thermodynamic data of each individual species. The equation of state is

$$
p=\sum_{i=\mathrm{i}}^{n} \rho_{i} \frac{\mathrm{R}_{0}}{w_{i}} \mathrm{~T},
$$

where $w_{i}$ represents the molecular weight, $\mathrm{T}$ represents the gas temperature, and $\dot{\omega}_{i}$ represents the species' specific mass production rate, which is dictated by the chemical reaction model.

The chemical model used in this study is a $\mathrm{C}_{2} \mathrm{H}_{2}-\mathrm{O}_{2}-$ diluent chemical mechanism for high-pressure ignition and detonation. ${ }^{42}$ This detailed chemistry model involves 21 elementary reactions, four of which are reversible, among the 15 species. Thermodynamic properties of the chemical species have been evaluated from the 9-coefficient NASA polynomial representation. ${ }^{43}$ The governing equations have been discretized on Cartesian uniform grids and solved with the DCD (Dispersion-Controlled Dissipation) scheme $^{44}$ with Strang splitting. To overcome the stiff problem, a sufficient number of sub-reaction steps are involved to assure the overall accuracy. ${ }^{45}$

Stoichiometric acetylene-oxygen-argon mixtures, i.e., $\mathrm{C}_{2} \mathrm{H}_{2}: \mathrm{O}_{2}: \mathrm{Ar}=2: 5: \mathrm{X}$, are used, in which X is determined by $\Phi$. The dilution ratio $\Phi$ is defined $\mathrm{X} /(2+5+\mathrm{X})$ and set to be $50 \%, 70 \%$, and $90 \%$ to represent different mixtures. ${ }^{35}$ The default inflow static pressure and temperature are set to 5.0 $\mathrm{kPa}$ and $298.15 \mathrm{~K}$, respectively. The slip reflecting boundary condition is used on the wedge surface, and the other boundaries are interpolated under the assumption of zero first-order derivatives for all flow parameters. The wedge angle is fixed to $25^{\circ}$ in all cases, and $M_{0}$ varies as a bifurcation parameter. On the lower computational boundary, the wedge typically starts from $x=1.28 \mathrm{~mm}$. Due to the multi-scale nature of the phenomena, both the computational domain and the mesh scale are adjusted and resolution studies are examined to ensure grid-independence. Initially, the whole flow field has uniform density, velocity, and pressure, which have been calculated according to $M_{0}$ and wedge angle. A previous study ${ }^{23}$ demonstrated that more complicated structures may appear in timedependent effects (e.g., in the structure building-up process), but this study focuses on steady structures and all the cases converge to their steady states.

\section{NUMERICAL RESULTS AND DISCUSSION}

\section{A. Wave structure and resolution study}

First of all, flow fields of ODW in the case of $M_{0}=8$, $\Phi=70 \%$ are simulated with the grid $32 \mu \mathrm{m}$ as shown in Fig. 3 . To illustrate wave structures, flow fields of four parameters, including pressure, temperature, total density, and species $\mathrm{OH}$ density, are plotted. The wave surface facing inflow is composed of two parts, a nonreactive shock upstream and an oblique detonation surface downstream, connected by a multi-wave point. Therefore, this flow field demonstrates an abrupt OSW-ODW transition, a structure that is typically

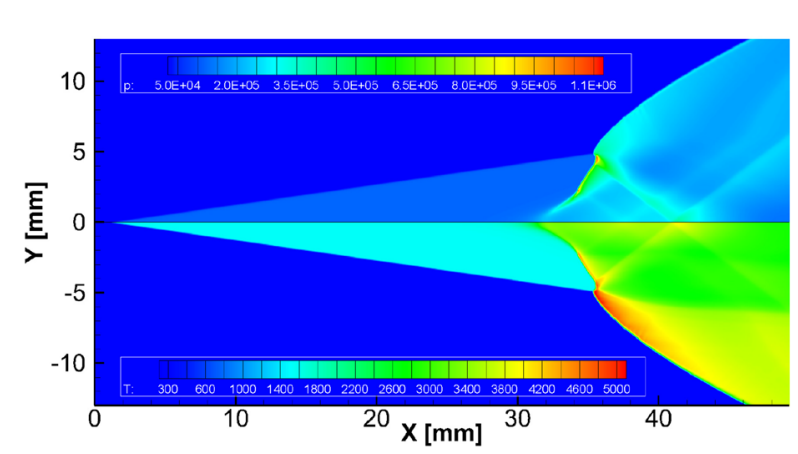

a

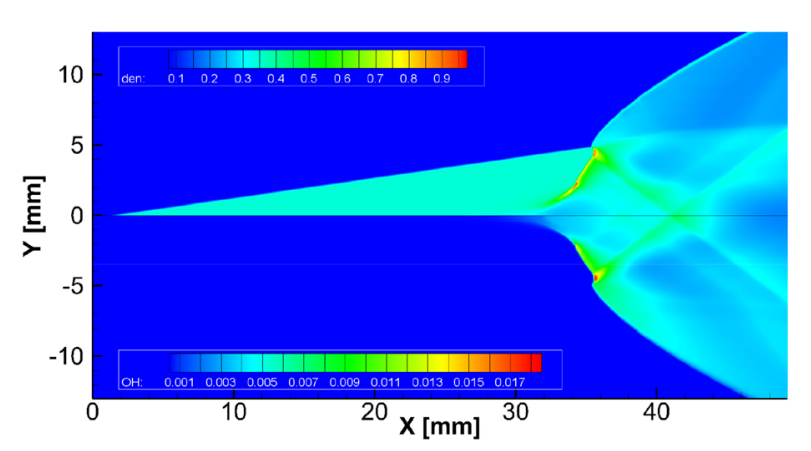

b

FIG. 3. Pressure [(a), upper], temperature [(a), lower], density [(b), upper], and species $\mathrm{OH}$ density [(b), lower] of ODW in the case of $M_{0}=8, \Phi=70 \%$.

observed in $\mathrm{H}_{2}$-air mixtures. However, this ODW differs from those sketched in Fig. 1, due to the initiation structure beneath the multi-wave point. As shown in Fig. 3, a reactive front faces the post-shock flow behind OSW, which can be easily distinguished in species $\mathrm{OH}$ density. Apparently, this front splits into two sections: the section near the wedge has a small oblique angle and induces a modest temperature increase; on the other side, the section away from the wedge has a large oblique angle and induces a strong temperature increase. Furthermore, across the reactive front, the density becomes low near the wedge but remains high away from the wedge. In general, a compression wave exists around the section near the wedge, while the secondary ODW around the section away from the wedge is detonation. Therefore, this initiation structure is actually a combination of both structures shown in Fig. 1.

To validate the effects of grid scale, the resolution study is performed by using the grid $16 \mu \mathrm{m}$. The results based on two grid scales are compared in Figs. 4 and 5, displaying the wave structure and temperature curves along the different $x_{-}^{-}$ axis parallel lines. Figure 4 shows that two wave structures are close to each other, illustrating a similar abrupt transition surface and two-section combined reactive front. Effects of fine grid are observed via formation of a triple point on the oblique detonation surface; however, this triple point moves downstream and will not affect the initiation region. On the other hand, the contact lines emanating from the reactive front strengthen when a fine grid is used, but the grid $32 \mu \mathrm{m}$ 


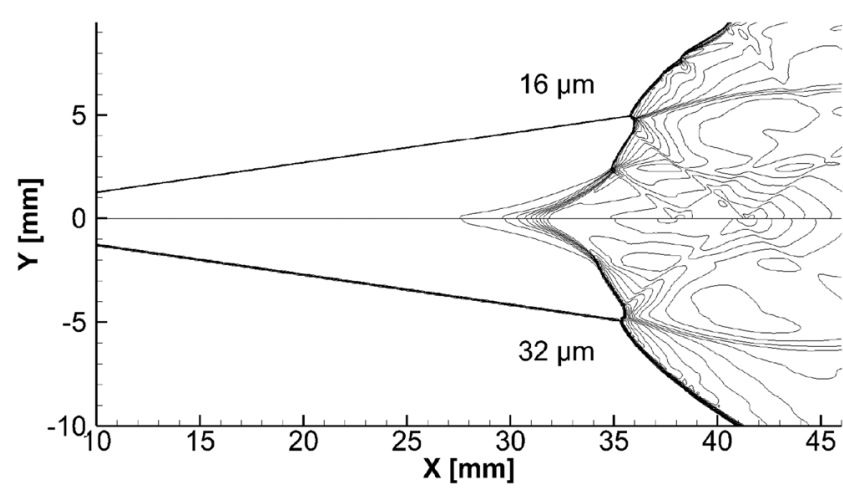

FIG. 4. Temperature of ODW with different grid scales in the case of $M_{0}=8$, $\Phi=70 \%$.

was found to be sufficient to capture them. The curves in Fig. 5 indicate the temperature variation along $\mathrm{x}$-axis parallel lines, which were chosen to be the wedge, the secondary ODW, and the main ODW surface. In general, the curves almost overlapped using different grids. To the secondary ODW $(y=3 \mathrm{~mm})$ and the main ODW surface $(y=6 \mathrm{~mm})$, there is a small position difference of about $0.5 \mathrm{~mm}$, derived from different grids. This difference is about $1.5 \%$ of the induction zone length of ODW on the whole, demonstrating that the effects of grid scale are neglectable here. Therefore, we use the grid $32 \mu \mathrm{m}$ to study the structural characteristics of the initiation region, and similar resolution studies have been performed for different cases in the following.

Keeping $\Phi=70 \%$, two other cases with different $M_{0}$ are simulated and shown in Fig. 6. In the case of $M_{0}=9$, abrupt transition appears, but the reactive front becomes simple. The two-section reactive front is replaced by one-section reactive front because the secondary ODW (the section coupled with oblique shock) disappears. Only the compression wave exists here, which differs from those of Fig. 3 . In the case of $M_{0}=10$ of Fig. 6(b), the wave structure evolves further and differs from both the cases above. Compared to the cast of $M_{0}=8$

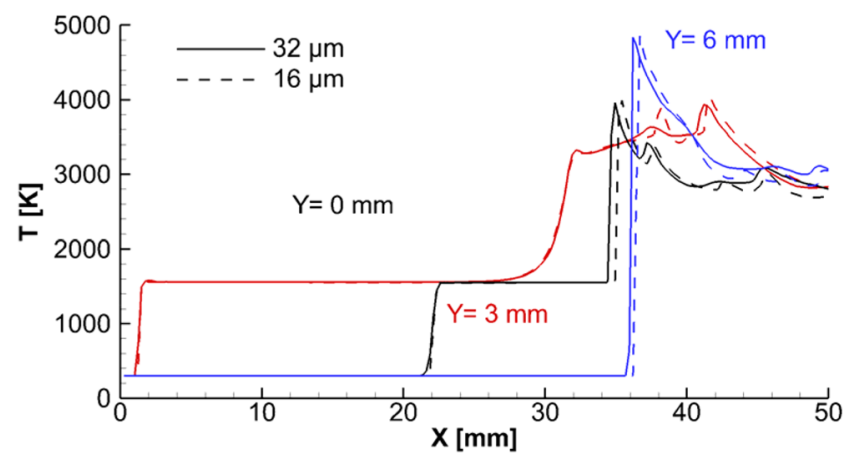

FIG. 5. Temperature curves along three $x$-axis parallel lines with different grid scales in the case of $M_{0}=8, \Phi=70 \%$.
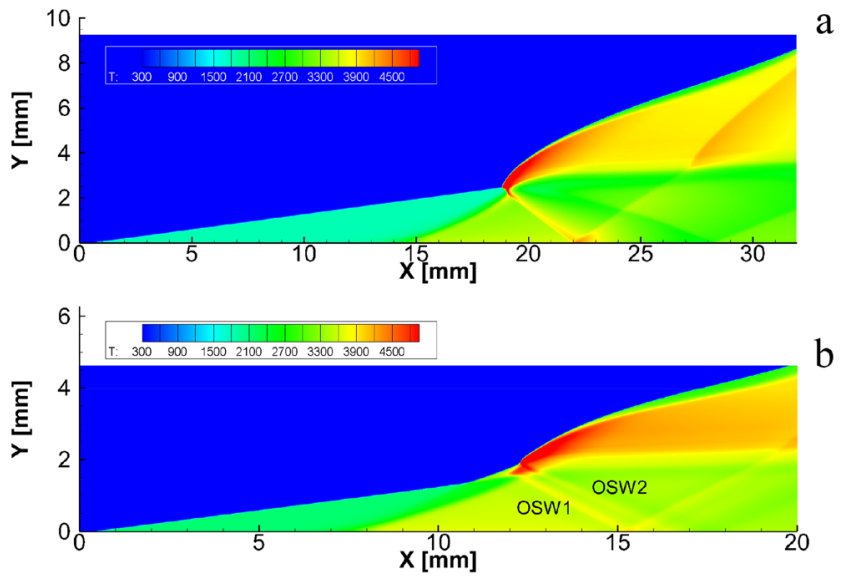

FIG. 6. Temperature of ODW in the cases of $\Phi=70 \%, M_{0}=9$ (a) and 10 (b).

(Fig. 3), not only the secondary ODW disappears, but the surface transition from OSW to ODW also becomes smooth. In addition to the disappearance of the secondary ODW, two oblique shocks, marked by ODW1 and ODW2, can be observed behind the reactive front. If the flow fields of the $M_{0}=8$ case are observed (Figs. 3 and 4), a similar two-shock structure appears. However, there are obvious differences between both cases. In the case of Fig. 3, a strong oblique shock emanated from the multi-wave point and a weak oblique shock emanated from the connection of the two-section reactive front. Nevertheless, as displayed in Fig. 6(b), the upstream oblique shock ODW1 is stronger than that of ODW2. Moreover, both shocks are very close in this case, which is often ignored.

It is not surprising that the structure of ODW is dependent on $\mathrm{M}_{0}$, which has been previously pointed out (e.g., Refs. 14-16). However, the two-shock structure around the reactive front, featured by two parallel oblique shocks in the detonation product, was not observed in previous $\mathrm{H}_{2}$-air mixtures and should therefore be associated with the complicated chemical reactions of $\mathrm{C}_{2} \mathrm{H}_{2}-\mathrm{O}_{2}-\mathrm{Ar}$. The relationship of the two-shock structure and the two-section reactive front seems complicated; however, the latter should be helpful for the formation of the former. Although the two-shock structure has never been reported to our knowledge, the twosection reactive front has been observed in $\mathrm{H}_{2}$-air mixtures. ${ }^{32}$ To ascertain why the two-shock structure forms and to study its mechanism, more cases are necessary.

\section{B. Effects of dilution ratio}

To further explore the structure in $\mathrm{C}_{2} \mathrm{H}_{2}-\mathrm{O}_{2}-\mathrm{Ar}$ mixtures, the dilution ratio $\Phi$, a key parameter on the property of combustible mixtures, is changed to $90 \%$ and $50 \%$. The results of $\Phi=90 \%$ cases are shown in Fig. 7 , demonstrating the smooth transition of OSW-ODW, regardless of the particular $\mathrm{M}_{0}$. According to a previous study, ${ }^{10}$ the transition type is sensitive to the difference of OSW and ODW angles. Increasing $\Phi$ results in a decrease in heat release and a relatively low angle of ODW surface. Thus, the angle difference becomes small and 

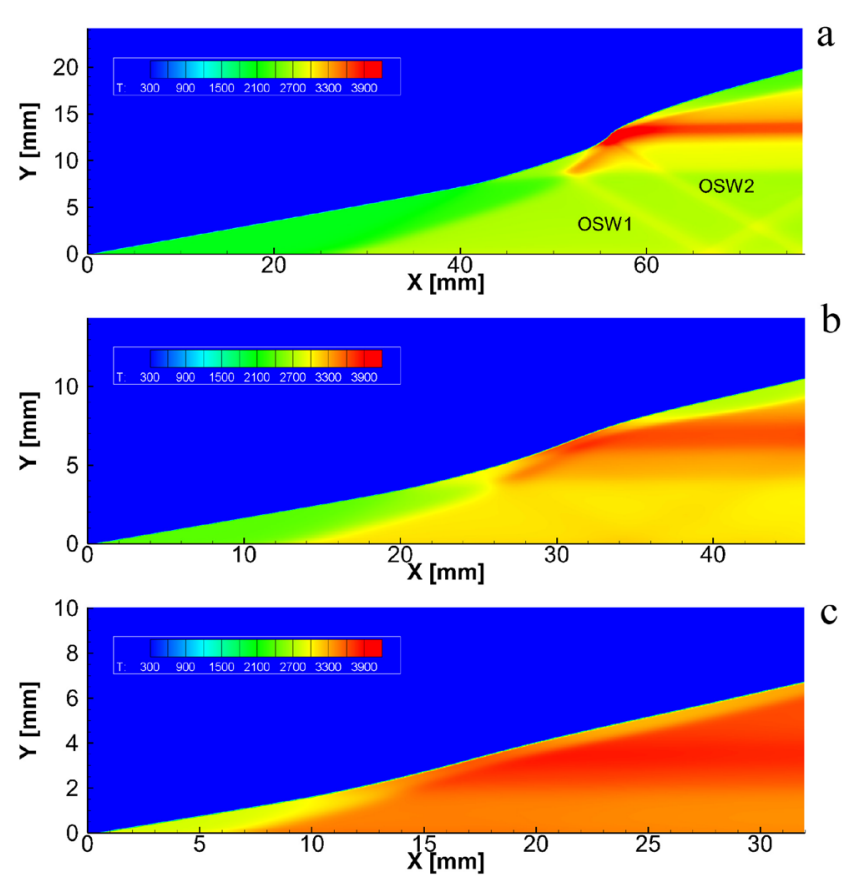

FIG. 7. Temperature of ODW in the cases of $\Phi=90 \%$ and $M_{0}=8(\mathrm{a}), 9$ (b), and 10 (c).

smooth transition appears in these high $\Phi$ cases. In general, these initiation structures are much simpler than those for the cases of $\Phi=70 \%$, especially when $M_{0}$ increases to 10 . However, the two-shock structure appearing in the case of $M_{0}=8$ can be observed, as shown in Fig. 7(a). The shocks here are relatively weak, and their distance is modest, not as close as that in the case of $\Phi=70 \%$ and $M_{0}=8$.

Figure 8 shows the ODW structures of $\Phi=50 \%$ cases. To demonstrate the results with further clarity, only the initiation

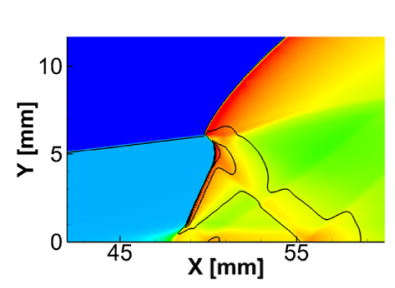

a
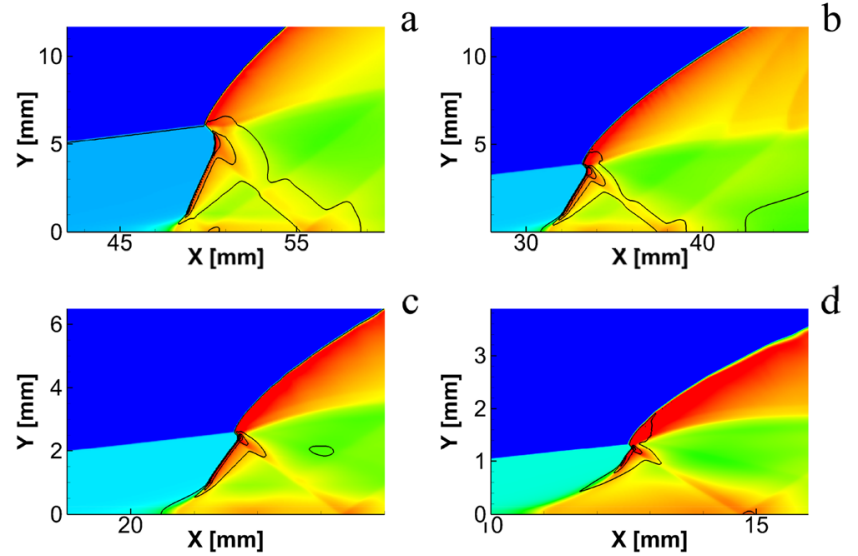

FIG. 8. Temperature (with black contours displaying pressure) of ODW in the cases of $\Phi=50 \%$ and $M_{0}=8(\mathrm{a}), 8.5(\mathrm{~b}), 9(\mathrm{c})$, and $10(\mathrm{~d})$. regions are displayed. All the ODW transitions are abrupt in contrast to the $\Phi=90 \%$ cases, but with the same mechanism, as discussed above. Clearly, the positions of initiation structures (and the angles of secondary ODWs) depend on $M_{0}$. With a low $M_{0}$, the angle of secondary ODW is large and the reactive front locates downstream, as shown in Fig. 8(a). By increasing $M_{0}$, the angle becomes small and the reactive front moves upstream [as shown from Figs. 8(a)-8(d)]. Moreover, the two-section reactive front becomes clear: the section coupled with the compression wave takes about $1 / 3$ of the whole reactive front in Fig. 8(c), while it takes over 1/2 in Fig. 8(d). Nevertheless, the oblique shocks originating from the connects of two sections are absent. This is similar to the structures in $\mathrm{H}_{2}$-air mixtures, but different from the structures in Fig. 3. Based on these results and previous results of $\mathrm{H}_{2}$-air mixtures, the formation of this two-shock structure is sensitive to the mixture, which is determined by the dilution ratio and the fuel type. The two-shock structure appears together with the two-section reactive front in Fig. 3; however, other cases demonstrate that either of them may appear without the other.

\section{Discussion on the structure features}

To demonstrate the structure, a sketch of ODW with twoshock structure is shown in Fig. 9. The reactive front, including the main ODW, is marked in red, which mainly illustrates the structure of Fig. 3. The front section near the wedge is coupled with the compression wave, which converges and results into a secondary ODW. In specific cases, the section of the compression wave may occupy the whole post-shock reactive front, evolving into the secondary ODW. By contrast, the secondary ODW cannot occupy the whole reactive front, and the compression wave part must appear, even taking a very short section, e.g., Fig. 8(a). The lengths of the compression wave and the secondary ODW depend on the $M_{0}$ and also their oblique angle. Two-shock structures may appear in the product, which extend downstream and interact with the wedge and slip line. Both shocks are almost parallel, and their strengths change case by case, determined by the interaction between the compression wave, oblique shock, and heat release.

To clarify the formation of the two-shock structure, it is necessary to explain why such a two-shock structure is not observed in Fig. 8(d). A very weak and short OSW appears in Figs. 8(a) and 8(b) near the wedge, whose effects are indicated by its interaction with another strong OSW in the

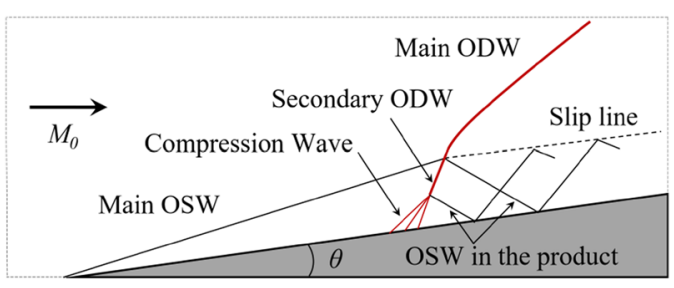

FIG. 9. Sketch of ODW with two-section reactive front. 
product. However, this shock disappears when the expansion wave elongates in Fig. 8(d), which differs from that in the case of $\Phi=70 \%$ and $M_{0}=8$. To explore the reason, Fig. 10 shows both pressure and temperature curves at different positions of both cases: $\Phi=70 \%, M_{0}=8$ and $\Phi=50 \%$, $\mathrm{M}_{0}=10$. The lines are chosen to approximately correspond the middle points of the compression wave and secondary ODW for each case. The numerical results demonstrate that in the case with a two-shock structure (i.e., $\Phi=70 \%, M_{0}=8$ ), the post-wave pressure behind the secondary ODW increases mildly and decays slowly, as shown in Fig. 10(a). Comparing the post-wave pressure behind the compression wave and the secondary ODW, a large high-pressure region exists, enabled by the formation of the other oblique shock. By contrast, in the case of $\Phi=50 \%$ and $M_{0}=10$ without two-shock, the postwave pressure behind the secondary ODW decays quickly, only generating a small high-pressure region. This provides a qualitative explanation for the formation of the two-shock structure, and further work is necessary in the future for a full clarification.

A further problem of the formation of two-shock structure is to explain cases without a two-section reactive front. Their flow fields are shown in the cases of $\Phi=70 \%, M_{0}=10$ [Fig. 6(b)] and $\Phi=90 \%, M_{0}=8$ [Fig. 7(a)]. An isolated, triangular reactive zone exists between two shocks for each case. Behind the OSW and ODW surfaces, the same inflow mixture usually results in different pressure and temperature. A oneshock wave configuration forms because the pressure behind
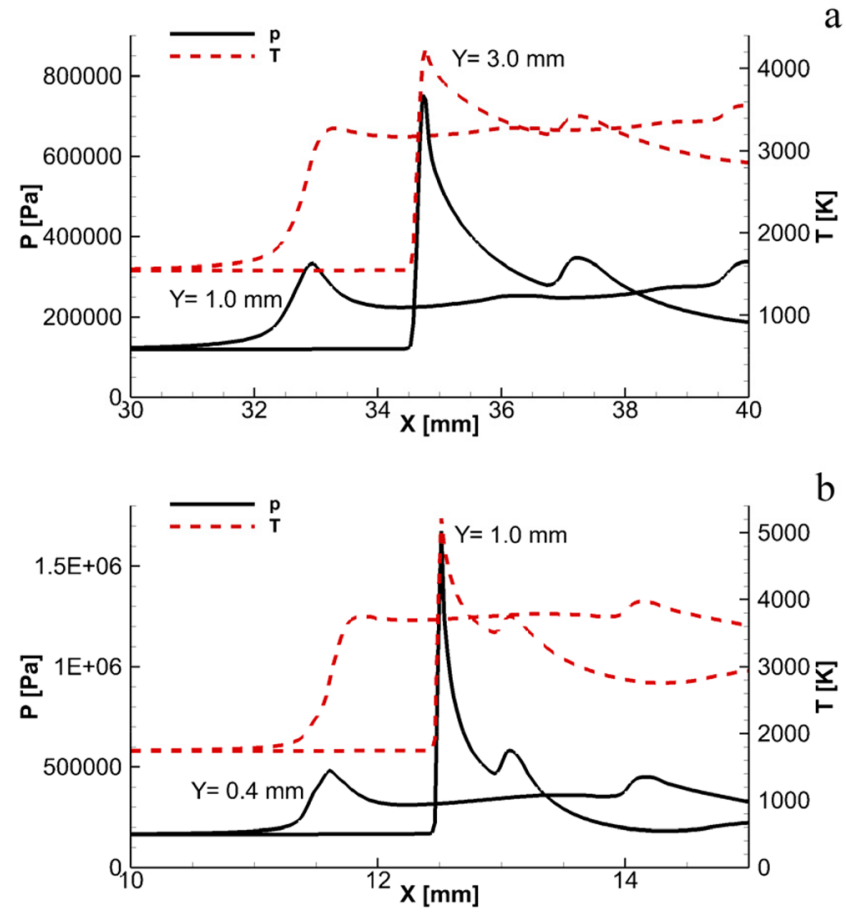

FIG. 10. Pressure and temperature along different lines for the cases of $\Phi=70 \%$, $M_{0}=8(\mathrm{a})$ and $\Phi=50 \%, M_{0}=10$ (b). the main ODW differs from the pressure behind the main OSW and the reactive front. When the reactive front splits into two sections, a two-shock structure forms, as shown in the case of $\Phi=70 \%, M_{0}=8$ (Figs. 3 and 4). Figures 6(b) and 7(a) enable the deduction that the isolated, triangular reactive zone cannot induce the same pressure as the region behind the main ODW; therefore, another shock is generated. In general, pressure matching of different regions of combustion product should dominate the formation of a two-shock structure, but further analysis of the wave configuration is necessary to further the understanding of this phenomenon.

It should be noted why no two-shock structure was observed in previous studies with global kinetic models (e.g., Refs. 16-18, 28). In one-step or two-step kinetic models, many parallel detailed chemical reactions are simplified as an irreversible heat release process and the variations of mixture properties are ignored. The specific heat capacity is usually set to be a constant, rather than a variable in detailed chemical reaction models. Considering that the two-shock structure only appears in the cases of $\Phi=70 \%$ and $90 \%$ in this study, the high specific heat capacity from Ar may play an important role. Effects of high specific heat capacity and different mixture properties should be further addressed to clarify the formation of the two-shock structure and to improve the understanding of ODW initiation.

\section{Discussion of characteristic lengths}

To quantify the features of ODW initiation, numerical and theoretical initiation lengths introduced in Ref. 32 are employed. The numerical initiation length is defined along the flow stream direction, parallel to the $x$-axis, from the wedge tip to the end of the induction zone, i.e., the location where the temperature increases above $10 \%$ along the flow lines. Correspondingly, a theoretical approach based on the constant volume combustion (CVC) theory is used to define the theoretical initiation length. The approach assumes an ideal post-oblique shock flow, where the mixture is completely burned, and both pressure build-up and the formation of pressure waves are weak near the wedge. Under these assumptions, calculations can be performed using the CHEMKIN package. ${ }^{43}$ First, the post-oblique-shock species densities and temperature are used to simulate $\mathrm{CVC}$ to obtain the reaction time required to attain a mixture temperature that achieves a $10 \%$ increase from its post-shock value. The theoretical initiation length is deduced by multiplying the time with the post-oblique-shock particle velocity. Despite its simple formulation, this analysis provides a predictive approach for the general structure of oblique detonations.

Both numerical and theoretical initiation lengths are shown in Table I and Fig. 11. For the cases of $\Phi=70 \%$ and $90 \%$ in Table I, the theoretical results are close to the corresponding numerical results, demonstrating that the mixture near the wedge is self-ignited by OSW compression. However, for the case of $\Phi=50 \%$, the deviation of numerical and theoretical results becomes significant when $M_{0}$ decreases, as shown in Fig. 11 and Table I. This means that the self-ignition cannot explain the initiation and other factors dominate the initiation process. A similar phenomenon is also observed in 
TABLE I. Numerical and theoretical initiation lengths of ODWs with different $\Phi$.

\begin{tabular}{|c|c|c|c|c|c|c|}
\hline \multirow[b]{2}{*}{$M_{0}$} & \multicolumn{2}{|c|}{$\Phi=50 \%$} & \multicolumn{2}{|c|}{$\Phi=70 \%$} & \multicolumn{2}{|c|}{$\Phi=90 \%$} \\
\hline & $\mathrm{L}_{\text {num }}(\mathrm{mm})$ & $\mathrm{L}_{\text {theo }}(\mathrm{mm})$ & $\mathrm{L}_{\text {num }}(\mathrm{mm})$ & $\mathrm{L}_{\text {theo }}(\mathrm{mm})$ & $\mathrm{L}_{\text {num }}(\mathrm{mm})$ & $\mathrm{L}_{\text {theo }}(\mathrm{mm})$ \\
\hline 8 & 45.0 & 3008 & 28.68 & 28.12 & 28.68 & 23.07 \\
\hline 9 & 19.33 & 22.02 & 12.99 & 12.34 & 12.99 & 10.88 \\
\hline 10 & 9.34 & 8.92 & 6.92 & 6.14 & 6.92 & 5.45 \\
\hline
\end{tabular}

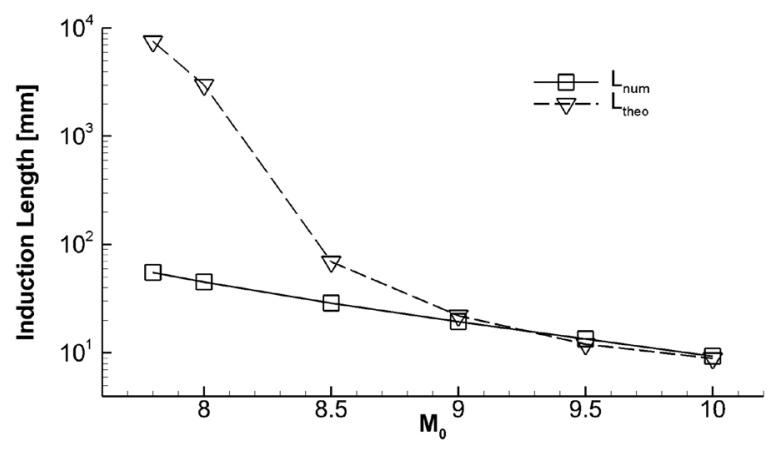

FIG. 11. Numerical and theoretical initiation lengths of ODWs with $\Phi=50 \%$.

$\mathrm{H}_{2}$-air mixtures, in which the former is named the kineticscontrolled initiation, and the latter the wave-controlled initiation. ${ }^{32}$ The initiation length of kinetics-controlled ODW can be predicted through CVC calculation, but for the latter initiation, it still lacks a rigid theory because only few cases were simulated and analyzed so far. In general, the initiation is kinetics-controlled except for cases with $\Phi=50 \%$ and a low $\mathrm{M}_{0}$ below 8.5 .

It is interesting to compare these results with those in $\mathrm{H}_{2}$-air mixtures. A previous study ${ }^{32}$ did not change the ratio of air or $\mathrm{N}_{2}$ but used a variable pressure, demonstrating that with stoichiometric $\mathrm{H}_{2}$-air mixtures, the static pressure does not change the initiation mechanism and for the low $M_{0}$ cases (of 7 and 8 in Ref. 32), the initiation is wave-controlled. This is similar to the case of $\Phi=50 \%$ in the present study but differs from cases with high $\Phi$. Therefore, the high temperature behind OSW is critical to induce the kinetics-controlled initiation, which is similar to a previous conclusion. For $\mathrm{C}_{2} \mathrm{H}_{2}-$ $\mathrm{O}_{2}$-Ar mixtures, Ar introduces a relatively high specific heat capacity; therefore, the cases with high $\Phi$ usually have a high post-oblique shock temperature, which results in a kineticscontrolled initiation.

\section{CONCLUSION}

Here, ODWs in stoichiometric acetylene-oxygen mixtures with $\Phi=50 \%-90 \%$ are simulated and studied. Based on the numerical results, the effects of $\Phi$ on the transition structure are studied for cases of different $M_{0}$. If $M_{0}$ changes between 8 and 10, the OSW-ODW transition is abrupt in cases of $\Phi=50 \%$, and smooth in cases of $\Phi=90 \%$. Either smooth or abrupt transition may appear in the case of $\Phi=70 \%$, depending on $\mathrm{M}_{0}$. A further study on initiation mechanism was performed by comparing the numerical initiation length with CVC calculation. The results demonstrate that the initiation is kinetics-controlled in the cases of $\Phi=70 \%$ and $90 \%$, but wave-controlled in the case of $\Phi=50 \%$ at a low $M_{0}$. Moreover, an initiation structure featured by a two-shock in the product was observed for the first time, whose formation can easily be understood when it appears with a two-section reactive front. The formation mechanism of the two-shock structure is discussed, and structures are analyzed by comparison to $\mathrm{H}_{2}$-air mixtures to clarify the effects of $\mathrm{C}_{2} \mathrm{H}_{2}-\mathrm{O}_{2}-\mathrm{Ar}$ mixtures.

\section{ACKNOWLEDGMENTS}

This work was supported by NSFC Nos. 11822202 and 91641130 .

\section{REFERENCES}

${ }^{1}$ G. D. Roy, S. M. Frolov, A. A. Borisov, and D. W. Netzer, "Pulse detonation propulsion: Challenges, current status, and future perspective," Prog. Energy Combust. Sci. 30, 545-672 (2004).

${ }^{2}$ P. Wolanski, "Detonation propulsion," Prog. Energy Combust. Sci. 34, 125-158 (2013).

${ }^{3}$ Y. Liu, W. Zhou, Y. Yang, Z. Liu, and J. Wang, "Numerical study on the instabilities in $\mathrm{H}_{2}$-air rotating detonation engines," Phys. Fluids 30, 046106 (2018).

${ }^{4}$ F. K. Lu, H. Fan, and D. R. Wilson, "Detonation waves induced by a confined wedge," Aerosp. Sci. Technol. 10, 679-685 (2006).

$\mathbf{5}_{\mathrm{J}}$. Urzay, "Supersonic combustion in air-breathing propulsion systems for hypersonic flight," Annu. Rev. Fluid. Mech. 50, 593-627 (2018).

${ }^{6}$ D. T. Pratt, J. W. Humphrey, and D. E. Glenn, "Morphology of standing oblique detonation waves," J. Propul. Power 7, 837-845 (1991).

${ }^{7}$ C. Li, K. Kailasanath, and E. S. Oran, "Detonation structures behind oblique shocks," Phys. Fluids 6, 1600-1611 (1994).

${ }^{8}$ C. Viguier, L. F. Figueira da Silva, D. Desbordes, and B. Deshaies, "Onset of oblique detonation waves: Comparison between experimental and numerical results for hydrogen-air mixtures," Symp. (Int.) Combust. 26, 3023-3031 (1996).

${ }^{9}$ L. F. Figueira da Silva and B. Deshaies, "Stabilization of an oblique detonation wave by a wedge: A parametric numerical study," Combust. Flame 121, 152-166 (2000).

${ }^{10} \mathrm{H}$. H. Teng and Z. Jiang, "On the transition pattern of the oblique detonation structure," J. Fluid Mech. 713, 659-669 (2012).

${ }^{11}$ T. Wang, Y. Zhang, H. Teng, Z. Jiang, and H. D. Ng, "Numerical study of oblique detonation wave initiation in a stoichiometric hydrogen-air mixture," Phys. Fluids 27, 096101 (2015).

${ }^{12}$ J. Y. Choi, E. J. R. Shin, and I. S. Jeung, "Unstable combustion induced by oblique shock waves at the non-attaching condition of the oblique detonation wave," Prog. Energy Combust. Sci. 32, 2387-2396 (2009). 
13J. Verreault, A. J. Higgins, and R. A. Stowe, "Formation and structure of steady oblique and conical detonation waves," AIAA J. 50, 1766-1772 (2012).

${ }^{14}$ J. Y. Choi, D. W. Kim, I. S. Jeung, F. Ma, and V. Yang, "Cell-like structure of unstable oblique detonation wave from high-resolution numerical simulation," Prog. Energy Combust. Sci. 31, 2473-2480 (2007).

$15 \mathrm{~J}$. Verreault, A. J. Higgins, and R. A. Stowe, "Formation of transverse waves in oblique detonations," Prog. Energy Combust. Sci. 34, 1913-1920 (2013).

${ }^{16}$ H. H. Teng, Z. L. Jiang, and H. D. Ng, "Numerical study on unstable surfaces of oblique detonations," J. Fluid Mech. 744, 111-128 (2014).

${ }^{17}$ P. Yang, H. D. Ng, H. Teng, and Z. Jiang, "Initiation structure of oblique detonation waves behind conical shocks," Phys. Fluids 29, 086104 (2017).

${ }^{18}$ Y. Zhang, L. Zhou, J. Gong, H. D. Ng, and H. Teng, "Effects of activation energy on the instability of oblique detonation surfaces with a one-step chemistry model," Phys. Fluids 30, 106110 (2018).

${ }^{19} \mathrm{Z}$. Ren, B. Wang, G. Xiang, and L. Zheng, "Effect of the multiphase composition in a premixed fuel-air stream on wedge-induced oblique detonation stabilisation," J. Fluid Mech. 846, 411-427 (2018).

${ }^{20} \mathrm{~S}$. Miao, J. Zhou, Z. Lin, X. Cai, and S. Liu, "Numerical study on thermodynamic efficiency and stability of oblique detonation waves," AIAA J. 56, 3112-3122 (2018).

${ }^{21} \mathrm{H}$. Teng, Y. Zhang, and Z. Jiang, "Numerical investigation on the induction zone structure of the oblique detonation waves," Comput. Fluids 95, 127-131 (2014).

${ }^{22}$ Y. Liu, Y. S. Liu, D. Wu, and J. Wang, "Structure of an oblique detonation wave induced by a wedge," Shock Waves 26, 161-168 (2016).

${ }^{23}$ Y. Liu, D. Wu, S. Yao, and J. Wang, "Analytical and numerical investigations of wedge-induced oblique detonation waves at low inflow Mach number," Combust. Sci. Technol. 187, 843-856 (2015).

${ }^{24} \mathrm{~B}$. Zhang and C. Bai, "Methods to predict the critical energy of direct detonation initiation in gaseous hydrocarbon fuels-An overview," Fuel 117, 294-308 (2014)

${ }^{25} \mathrm{~B}$. Zhang, "The influence of wall roughness on detonation limits in hydrogen-oxygen mixture," Combust. Flame 169, 333-339 (2016).

${ }^{26}$ Z. Pan, B. Fan, X. Zhang, M. Gui, and G. Dong, "Wavelet pattern and selfsustained mechanism of gaseous detonation rotating in a coaxial cylinder," Combust. Flame 158, 2220-2228 (2011).

${ }^{27}$ Z. Pan, J. Qi, J. Pan, P. Zhang, Y. Zhu, and M. Gui, "Fabrication of a helical detonation channel: Effect of initial pressure on the detonation propagation modes of ethylene/oxygen mixtures," Combust. Flame 192, 1-9 (2018).

${ }^{28}$ P. Yang, H. Teng, H. D. Ng, and Z. Jiang, "A numerical study on the instability of oblique detonation waves with a two-step induction-reaction kinetic model," Proc. Combust. Inst. 37, 3537 (2019).

${ }^{29} \mathrm{P}$. Yang, H. Teng, Z. Jiang, and H. D. Ng, "Effects of inflow Mach number on oblique detonation initiation with a two-step induction-reaction kinetic model," Combust. Flame 193, 246-256 (2018).
${ }^{30}$ Y. Fang, Z. Hu, H. Teng, Z. Jiang, and H. D. Ng, "Effects of inflow equivalence ratio inhomogeneity on oblique detonation initiation in hydrogen-air mixtures," Aerosp. Sci. Technol. 71, 256-263 (2017).

${ }^{31} \mathrm{~K}$. Iwata, S. Nakaya, and M. Tsue, "Wedge-stabilized oblique detonation in an inhomogeneous hydrogen-air mixture," Prog. Energy Combust. Sci. 36, 2761-2769 (2017).

${ }^{32} \mathrm{H}$. Teng, H. D. Ng, and Z. Jiang, "Initiation characteristics of wedgeinduced oblique detonation wave in a stoichiometric hydrogen-air mixture," Prog. Energy Combust. Sci. 36, 2735-2742 (2017).

${ }^{33}$ J. H. S. Lee, "Dynamic parameters of gaseous detonations," Annu. Rev. Fluid Mech. 16, 311-336 (1984)

${ }^{34}$ M. I. Radulescu, H. D. Ng, J. H. S. Lee, and B. Varatharajan, "The effect of argon dilution on the stability of acetylene/oxygen detonations," Prog. Energy Combust. Sci. 29, 2825-2831 (2002).

${ }^{35}$ B. Zhang, N. Mehrjoo, H. D. Ng, J. H. S. Lee, and C. Bai, "On the dynamic detonation parameters in acetylene-oxygen mixtures with varying amount of argon dilution," Combust. Flame 161, 1390-1397 (2014).

${ }^{36} \mathrm{~S}$. Maeda, J. Kasahara, and A. Matsuo, "Oblique detonation wave stability around a spherical projectile by a high time resolution optical observation," Combust. Flame 159, 887-896 (2012).

${ }^{37}$ S. Maeda, S. Sumiya, J. Kasahara, and A. Matsuo, "Initiation and sustaining mechanisms of stabilized oblique detonation waves around projectiles," Prog. Energy Combust. Sci. 34, 1973-1980 (2013).

${ }^{38} \mathrm{C}$. Li, K. Kailasanath, and E. S. Oran, "Effects of boundary layers on oblique detonation structures," AIAA Paper No. 93-0450, 1993.

${ }^{39}$ C. Huete, A. L. Sánchez, F. A. Williams, and J. Urzay, "Diffusion-flame ignition by shock wave impingement on a supersonic mixing layer," J. Fluid Mech. 784, 74-108 (2015).

${ }^{40}$ C. Huete, A. L. Sánchez, and F. A. Williams, "Diffusion-flame ignition by shock-wave impingement on a hydrogen-air supersonic mixing layer," J. Propul. Power 33, 256-263 (2017).

${ }^{41}$ Z. Ren, B. Wang, and L. X. Zheng, "Numerical analysis on interactions of vortex, shock wave, and exothermal reaction in a supersonic planar shear layer laden with droplets," Phys. Fluids 30, 036101 (2018).

${ }^{42}$ B. Varatharajan and F. A. Williams, "Chemical-kinetic descriptions of hightemperature ignition and detonation of acetylene-oxygen-diluent systems," Combust. Flame 124, 624-645 (2001).

${ }^{43}$ B. J. McBride, M. J. Zehe, and S. Gordon, "NASA Glenn coefficients for calculating thermodynamic properties of individual species," Report No. NASA/TP-2002-211556, NASA Glenn Research Center, Cleveland, USA, 2002.

${ }^{44} \mathrm{Z}$. Jiang, "On dispersion-controlled principles for non-oscillatory shockcapturing schemes," Acta Mech. Sin. 20, 1-15 (2004).

${ }^{45} \mathrm{H}$. C. Yee, D. V. Kotov, W. Wang, and C. W. Shu, "Spurious behavior of shock-capturing methods by the fractional step approach: Problems containing stiff source terms and discontinuities," J. Comput. Phys. 241, 266-291 (2013). 